\title{
Intelligent Processing of Experimental Data in ISES Remote Laboratory
}

\author{
http://dx.doi.org/10.3991/ijoe.v12i03.5538 \\ M. Gerža ${ }^{1}$, F. Schauer ${ }^{1,2}$ \\ ${ }^{1}$ Tomas Bata University in Zlín, Zlín, Czech Republic \\ ${ }^{2}$ Trnava University in Trnava, Slovak Republic
}

\begin{abstract}
The paper deals with the design of the intelligent processing of experimental data measured in the remote laboratories, where the measurements are performed by connected clients via the finite-state machine residing at an advanced server. As physical hardware and software is used the Internet School Experimental System (ISES). This platform is intended for educational purposes at schools and universities to provide the suitable measuring environment for students using computers. It is especially suitable for distance students who cannot attend regular courses during their studies of physics, chemistry or electro-engineering.
\end{abstract}

At present, the remote laboratory does not have any data processing technique. This results in excessive demands on storing capacity. The suggested solution solves this drawback by processing and archiving techniques to store measured data after their organizing in predetermined structures and concomitant data volume reduction.

The paper is organized in the following manner. In the first section, Introduction, the ISES remote laboratory concept is introduced, following, State of the art, describing present stage of its ISES physical hardware development. The description of ISES physical software is concentrated at the Measureserver core unit realized as the finite-state machine. The next section deals with the design and implementation of the intelligent data processing component, which is focused on the extracting, filtering and archiving of measured data coming from the Measureserver unit communicating with a physical experiment. Finally, all the functional benefits are summarized for interested, who are involved in the remote experiments construction.

Index Terms-ISES, Measureserver, finite-state machine, remote laboratory, physical experiment, analysis.

\section{INTRODUCTION}

The traditional teaching methods for students at schools and universities are obsolete and not effective enough. The students often expect higher level teaching methods in the field of physics, biology, chemistry and electroengineering, which can help them to perceive real world phenomena in a better way. The problem is also an accessibility of the educational materials, especially for distant students who nowadays prefer studying scientific topics using their computers via the Internet. These problematic points are effectively solved by the remote laboratories (RLs) so called e-laboratories. The RLs built on the ISES platform are developed by the RL Consortium (Charles University in Prague, Tomas Bata University in Zlín and Trnava University in Trnava) for educational purposes.
The ISES is an advanced tool for real-time operation, data acquisition, data processing, controlling hardware (HW) and visualization.

It is an open system consisting of the ISES hardware and software intended for hands-on experiments called ISES WIN. However, there is also an alternative for using remote experiments (REs), the ISES WEB Control Kit. Both types of the experiments are built as the burst (fast) and normal (slow) to offer students a wider spectrum of knowledge. The basic version of ISES RLs has been developed by Charles University in Prague. When the ISES RL became a standard educational tool, it was significantly improved to a higher level tool by Tomas Bata University in Zlín in cooperation with Charles University in Prague implemented as the new user environment, EASY REMOTE-ISES, in order to simply building REs by laymen [4].

Let us describe the ISES RL concept. It consists of five units as the installed HW (convertor, universal control board, physical modules categorized as meters, sensors and devices), Measureserver, Imageserver, Webserver and Webclient. More technical details and deployed applications are available in [1][2][5][6][7] and [8]. The ISES RLs were recently integrated into a new platform called Remote Laboratory Management System (REMLABNET). It is a platform providing advanced autonomous units as services to the ISES RL administrators and registered clients. The schematical arrangement of all the involved units and corresponding communication is presented in Figure 1.

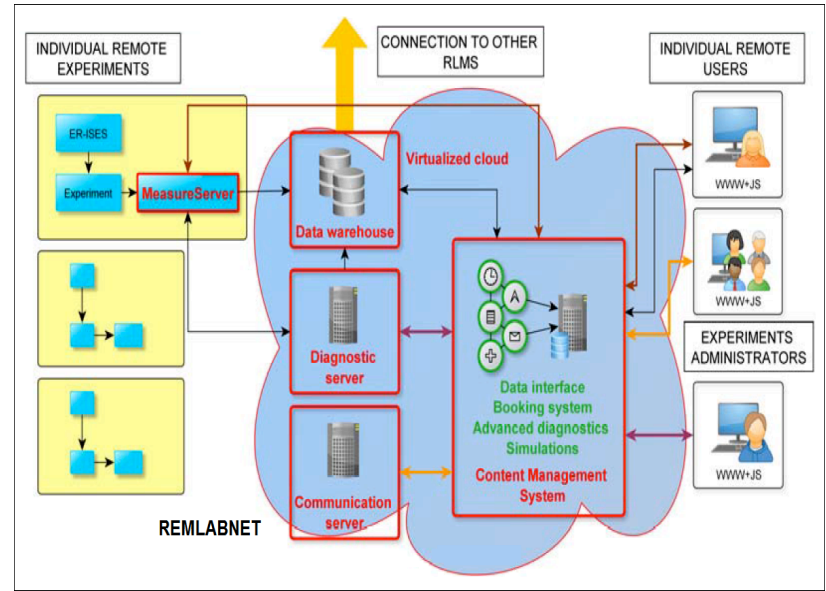

Figure 1. Schematical arrangement of the REMLABNET platform including ISES remote laboratories providing a set of the physical experiments (left) and connected clients (right) [3] 


\section{STATE OF THE ART}

The ISES RL units internally exploit appropriate controlling, processing and communication functions to cooperate with other autonomous units in order to deliver measured data and metadata.

\section{A. Physical hardware}

Basically, there are two concepts implemented for the ISES laboratories - local and remote, built on the same physical HW. The ISES is a modular platform based on the three standard parts. As the first part, it is the $\mathrm{AD} / \mathrm{DA}$ (Analog-to-Digital/Digital-to-Analog) convertor installed as the PCI 1202 interface card inside a control computer. Further parts are the ISES universal control board and a set of various sensors for physics, chemistry and biology. The platform offers the possibility of simultaneous measuring and data displaying for 8 input analogue channels. There are also available 2 analogue and 2 binary output channels. The analogue output channels work as programmable voltage sources. Maximum sampling frequency is $100 \mathrm{kHz}$ enabling to study sounds or other periodicity signals. A standard set of the physical HW available is illustrated in Figure 2.

\section{B. Measureserver unit}

The Measureserver (MS) unit is a significant software part of the ISES RL concept. It is an advanced communication server between the physical HW and remote clients (students). The MS core is designed as a finite-state machine (FSM) for a setup of logical functions solving prescribed activities. Its functioning is drawn from the concise PSC script file.

With respect to the physical HW, the MS in reality communicates with a software driver of the PCI 1202 interface card. This process is digital entirely based on reading data (values) directly from particular pins and writing data to respective pins which are translated by the AD/DA convertor. These pins are perceived as the inputs and outputs located on the universal control board that allows connecting particular ISES physical modules like meters, sensors and devices into the system.

The data (specific commands) come from the remote client to MS. The communication is realized by standard protocols via the Internet. The commands directly go to the REMLABNET where the client can find a desired ISES RL in a database to handle its results.

All commands, incoming from the client and physical $\mathrm{HW}$, are processed in a deterministic way by FSM realized by the two parsers. The first is the LR(1) parser that processes commands from the configuration file (CFG) for the purpose of the GUI (graphical user interface) settings. This parser is based on the static state transition tables called parsing tables, which codify the language grammar. The parsing tables are parameterized together with a lookahead terminal. The lookahead establishes the maximum incoming tokens, the parser can use to decide, which rule it should use.

The second one is the Recursive descent parser (RDP) that processes commands (instructions) coming from the PSC script file to create the data structures and logic for the RE. It uses a general form of top-down parsing where

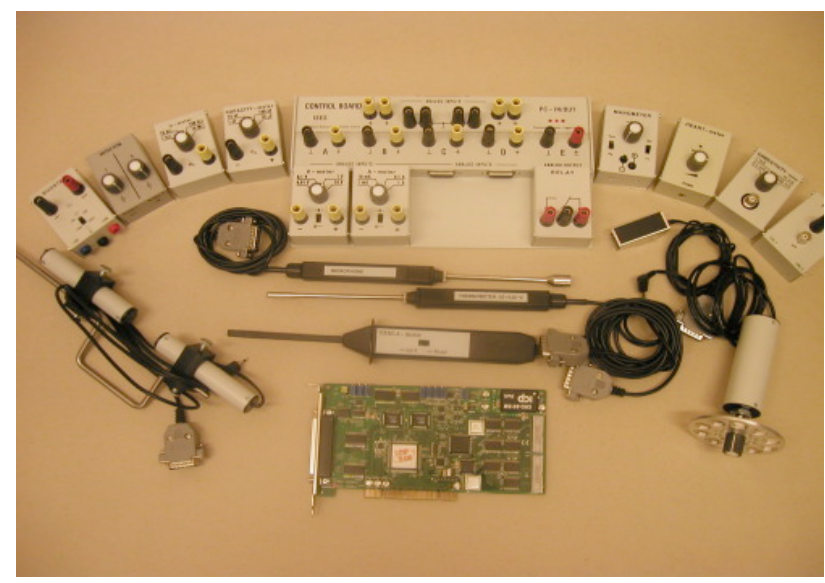

Figure 2. ISES physical hardware including the PCI 1202 interface card and a broad range of the involved meters, sensors and devices [6]

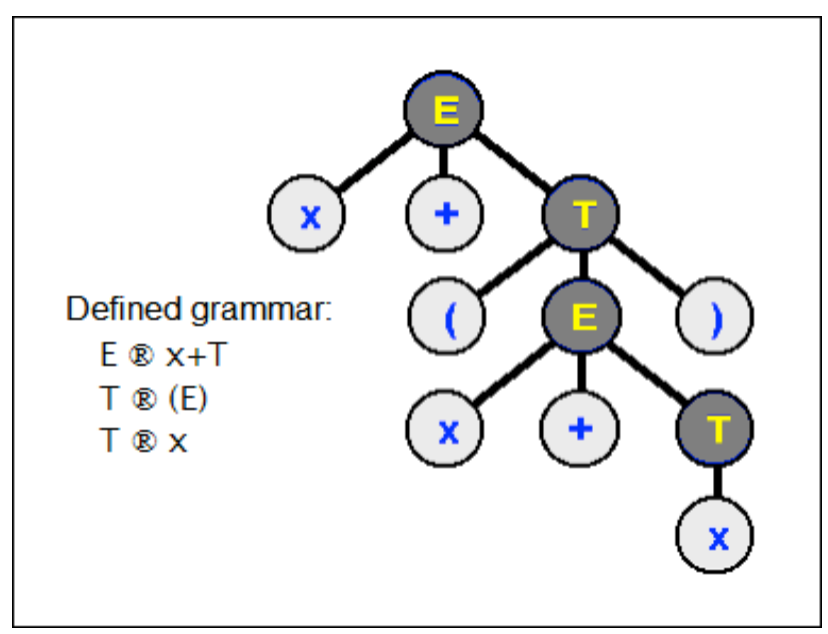

Figure 3. Example of the derivation tree for $x+(x+x)$ by using the Recursive Descent Parser working inside the Measureserver unit [9]

backtracking may be involved. The parsing algorithm is based on the walking through a tree. An example with the defined grammar is shown in Figure 3.

\section{Webserver unit}

The Webserver unit provides the Nginx services coming into the process when client enters a web page of the ISES RL via the REMLABNET platform. Nginx is an open source reverse proxy server for HTTP, HTTPS, SMTP, POP3 and IMAP protocols and a web server.

\section{Webclient unit}

The Webclient unit is a comfortable graphical interface allowing registered clients to access either the REMLABNET or EU RLMS Go-Lab platforms. Clients are connected to given RLs with additional services provided by the respective platform.

Necessary web pages are provided by the RL server, nowadays with the diagnostic module, reservation system and class formation. A well-arranged design of the RE web pages and serviceability play an important role for experimenting as shown in Figure 4 that illustrates the deployed RE "Free fall in a tube with vacuum". 


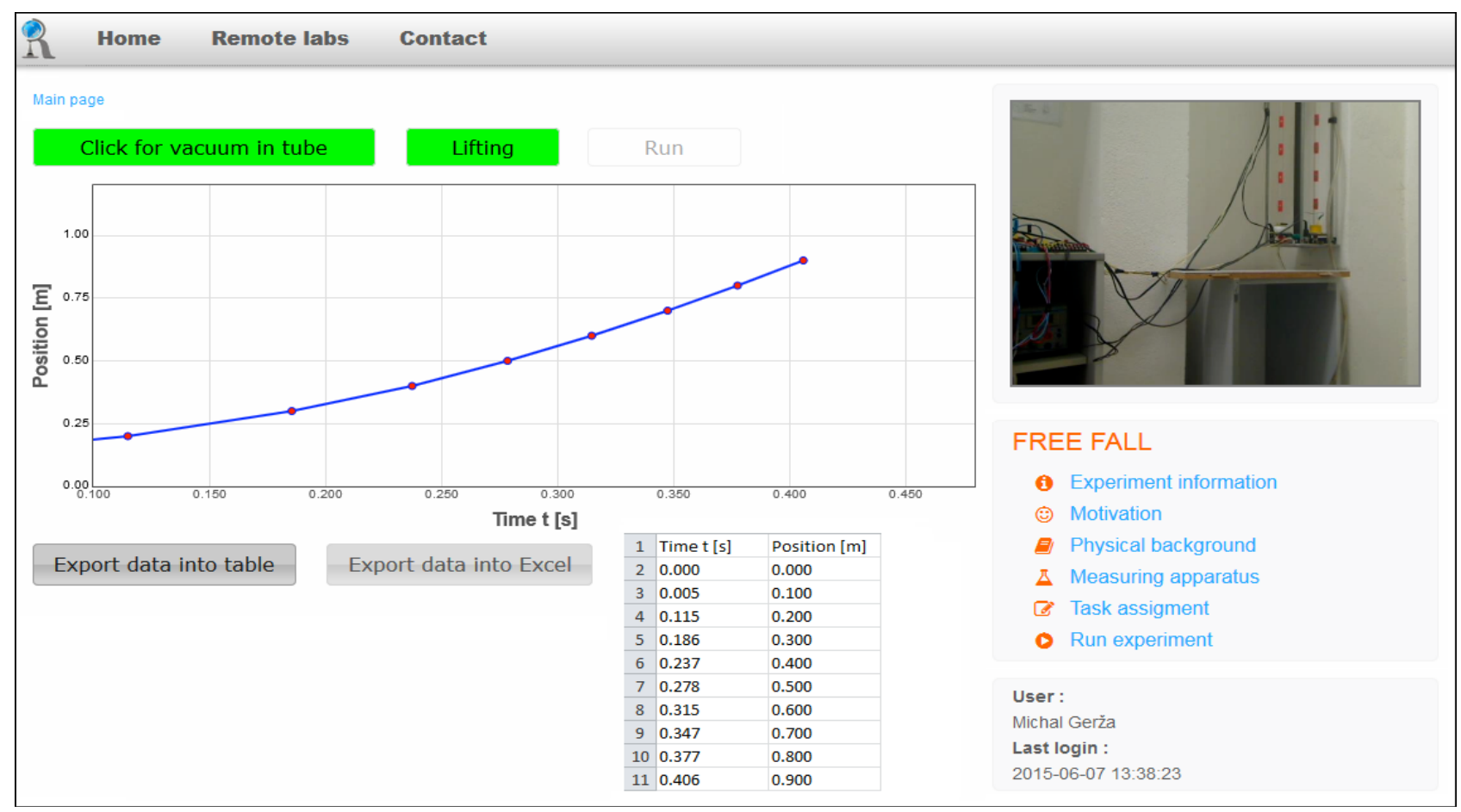

Figure 4. Client's web page of the ISES RE "Free fall in a tube with vacuum" provided by the REMLABNET platform [3]

TABLE I.

THE COMMANDS FOR A COMPLETION OF OPERATIONS TO PROVIDE EXTRACTED AND FILTERED DATA COMING FROM THE REMOTE EXPERIMENT

\begin{tabular}{|l|l|}
\hline \multicolumn{1}{|c|}{ Command } & \multicolumn{1}{c|}{ Description } \\
\hline CMD_BURST_EXPERIMEN & Provides all measured data from the fast RE \\
\hline CMD_START_EXPERIMENT & Provides metadata from the normal RE when it started running \\
\hline CMD_STOP_EXPERIMENT & Provides metadata from the normal RE when it stopped running \\
\hline CMD_READ_EXPERIMENT & Provides a set of specific measured data from the normal RE when a client requires \\
\hline CMD_RUNNING_EXPERIMENT & Provides current measured data from the normal RE \\
\hline CMD_LIST_EXPERIMENTS & Provides a list of all the running REs \\
\hline CMD_GET_SRC_DEV_HANDLE & Provides a list of the writing devices \\
\hline CMD_GET_DST_DEV_HANDLE & Provides a list of the reading devices \\
\hline CMD_GET_QUENE & Provides a list of the connected clients \\
\hline CMD_GET_QUEUE_POSITION & Provides a position of the active client \\
\hline CMD_LIST_DEVICES & Provides a list of all the involved devices \\
\hline CMD_READ_FAST_LOG & Provides measured high frequency data from the selected device \\
\hline CMD_READ_LOG & Provides measured data from the selected device \\
\hline CMD_GET_DEV_VALUE & Read a value from the selected device \\
\hline CMD_SET_DEV_VALUE & Writes a value to the selected device \\
\hline
\end{tabular}

\section{INTELLIGENT DATA PROCESSING}

The section deals with the design of the intelligent data processing component (IDPC) used for obtaining measured data from the RE. It became an integral part of the MS for the purpose of cooperating with the RDP. The reason of this design is an absence of extracting, filtering and archiving of experimental data in the ISES RL.

\section{A. Data extraction process}

The ISES RL produces experimental data delivered by installed ISES sensors measuring e.g. voltage, current or temperature in order to elucidate phenomena observed by the clients. These data are continuously accumulated and processed in the MS to store and forward the measured data, describing the phenomenon, to clients for an analysis. The existing ISES RL concept provides very rich data containing both phenomenon data and data describing executing experiment in general. Part of these data are very important from didactical and pedagogical reasons (so called LOG file), describing the client's behavior while measuring is worth evaluation. The output of this procedure may be used for RL administrators and involved teachers to optimize design and functioning. All commands needed for specific operations of extracting and filtering are closely listed in Table 1.

The commands are received via the WebSocket protocol (providing full-duplex communication channels over a single TCP connection), which was newly implemented into the MS to cooperate with JavaScript widgets placed 
on the web page. The MS listening socket waits for an incoming packet including the appropriate command identifier at the first position to find the data structure in order to read the measured data. As the next step, the relevant case is activated to read metadata and structured measured data in the internal buffers inside the MS. At this moment, the IDPC starts working on the filtering through the use of SQL queries. A client can request, e.g. data from two sensors of the five installed ones in the chosen time interval. An example of the SQL query is shown in Figure 5 to obtain measured data provided to the client for an analysis.

\section{B. Data archiving process}

The IDPC goes in for archiving the whole structured file into the XML (Extensible Markup Language) format, which defines a set of rules for encoding of our data in a format, both human and machine readable. The identical data structure of every RE is created for easy archiving and dispatching. The Microsoft XML Core Services (MSXML) module was used for the purpose of the XML structure forming, data accumulating and files archiving. In our case, we exploited the MSXML version 3.0 implemented inside the MS core.

The archiving process consists of the following two steps. When the client's request comes in, the IDPC immediately activates a relevant case to perform the filtering by incoming commands. After the filtering process, the XML structure forming starts to create the fixed XML file as shown in Figure 6.

Resulting data XML format is created in two different ways, depending on a character of the RE, either continuously for slow processes or using accumulation buffers for fast processes. After the completion of archiving, a unique file is generated and saved to a hard disk of the RE server and dispatched to the data warehouse of the REMLABNET for future purposes. The final dispatching phase is an optional feature configured by administrators as shown in Figure 7.

Every executed measurement is archived to a unique XML file consisted of the date, time and two basic identifiers. Related to the normal RE, when a client presses the start button, the recording begins until the stop one is pressed. If the burst RE runs, a client presses the start button only to make the data record. At this moment, the filtering, archiving and optional dispatching processes are activated in successive steps inside the IDPC. A typical example of the XML file, including identifiers and attributes, is listed in Figure 8.

\section{Data analyzing process}

The subsequent data analysis is often required with the goal of an elucidation of both pedagogical or psychological aspects of respective RE and its improvement using the data mining techniques. It constitutes the process of extracting nontrivial and potentially useful information from the big data providing various diagnoses, classification or forecasting problems. These techniques identify trends, which go beyond the simple analysis. For this purpose the techniques like the Gaussian mixture models, regression algorithms, decision trees, association rules and neural networks are used. The analysis is entirely executed in the data warehouse.

The final results of such sophisticated analysis are exploited by the RE designers, teachers and psychologists to

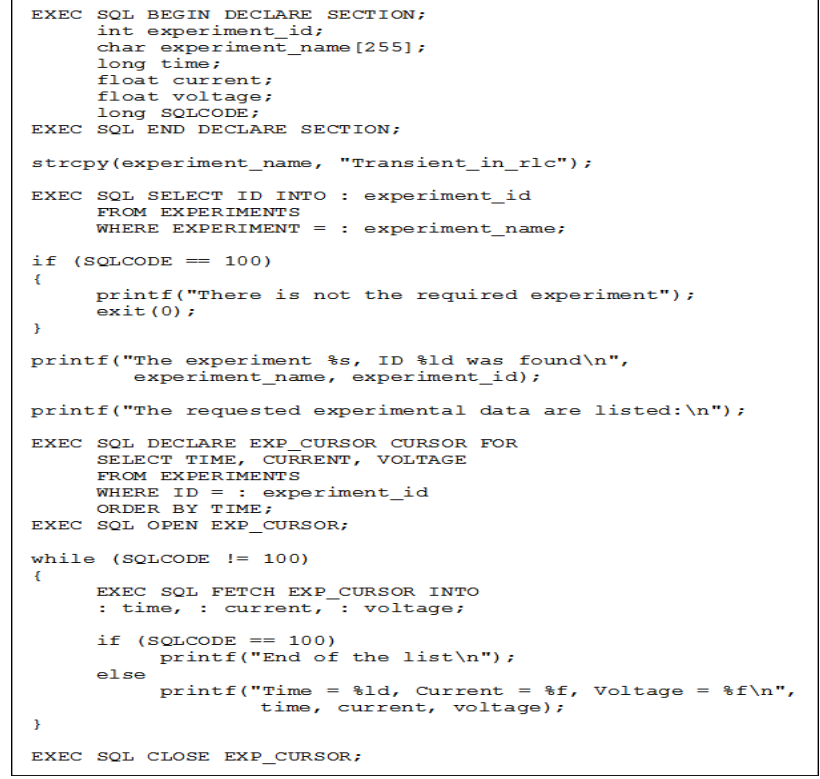

Figure 5. Example of the SQL query presents the filtering process implemented by $\mathrm{C}$ programming language to acquire the measurement time and the experimental data from an amperemeter (current) and voltmeter (voltage), whose historical data reside inside the MS unit

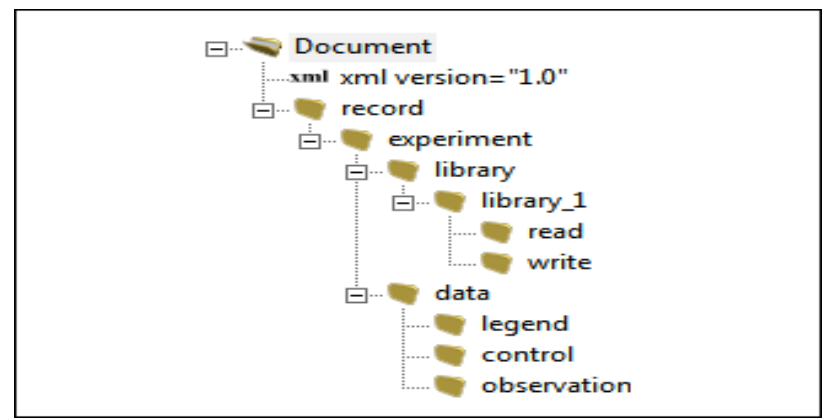

Figure 6. Data structure of archived RE based on the XML concept

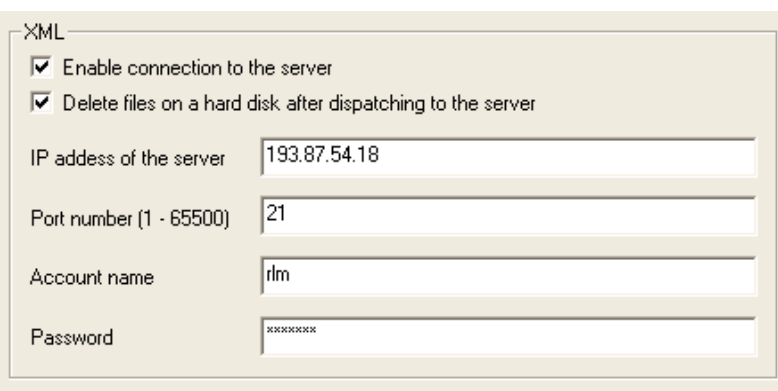

Figure 7. Dialog box used for a configuration of the dispatching feature to deliver archived XML files to the remote data warehouse

improve REs in the direction of more effective educational approach based on the ISES RL concept.

\section{Data publishing process}

A certain segment of the data collected is also provided to other clients registered to the REMLABNET platform to be employed for their respective analysis and publications. Another advantage of data releasing is to reduce measurement overhead accumulated by another client for saving time and comparative purposes. According to our running practice, all the data are stored in a database in the data warehouse. 


\section{FunCTIONAL BENEFITS}

The IDPC features offer a more comfortable data management. As the first benefit, the RE designers and involved teachers can easily analyze the measured data and clients behavior during their experimentation. The second benefit seems to be exposing a new databaseprovided by the data warehouse to registered clients who can exploit existing measured records produced by previous clients. The reason of this implementation was the absence of the data archiving process and intelligent analysis required for routine educational purposes. The detailed scheme of all the MS components including a position of the IDPC is shown in Figure 9.

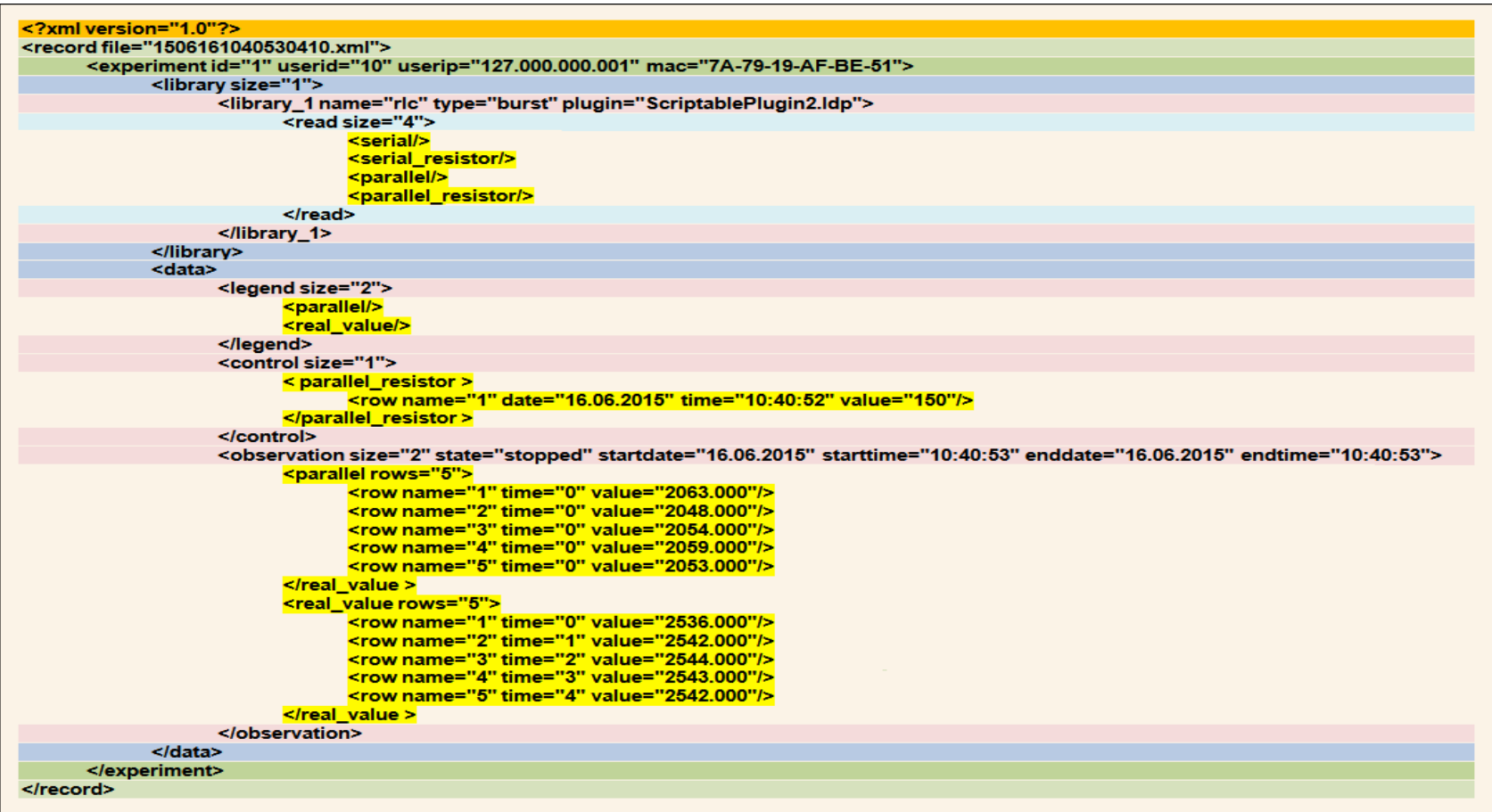

Figure 8. Example of the XML structure built for archiving of the experimental data received from the RE where the coupled colored lines present particular subsections (identical colored line pairs indicate subsections at the same level), yellow records show measured data sets obtained from the RE "Electric and electromagnetic phenomena in RLC circuits with the variable damping"

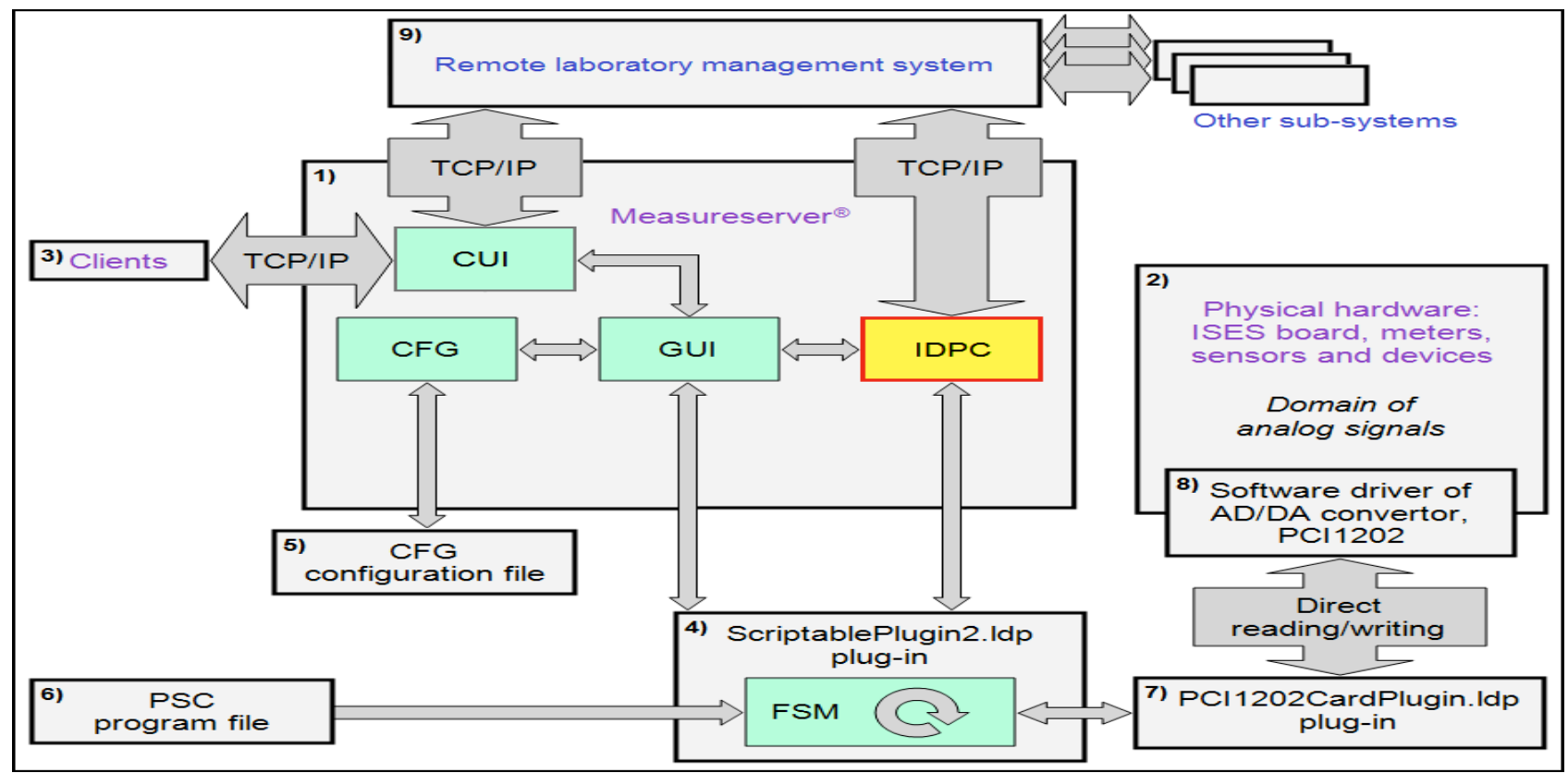

Scheme of communication relationships among particular components cooperating with the Measureserver unit as follows:

Figure 9. 1) Measureserver including the initial configuration (CFG), graphical user interface (GUI) and new intelligent data processing component (IDPC), 2) ISES physical modules set building the RE, 3) Clients connected via the RE web page, 4) Plug-in building the RE logic by the finite-state machine (FSM), 5) Configuration file delivering the initial parameters, 6) Control program file creating the RE structures, logic states and communication, 7) Plug-in communicating with the PCI 1202 AD/DA converter driver, 8) PCI 1202 AD/AD converter driver, 9) REMLABMET platform integrating, maintaining and monitoring all the ISES RLs located in different places 


\section{CONCLUSIONS}

The paper presented the intelligent data processing of experimental data collected from the ISES remote laboratory. We focused on the design and implementation of the data processing component used for the extraction, archiving, analyzing and publishing of measured and operational data obtained from the finite-state machine running inside the Measureserver unit.

The data extracting, data filtering, prescript structure storing, XML files creating and files dispatching into the warehouse were described. It comes then to advanced operations for the analysis exploiting some artificial intelligence methods to gain substantial information mainly intended for the ISES remote experiments improvement, pedagogical and didactical purposes.

We have formulated the following conclusions.

1. The experimentation provided by the ISES remote laboratory is a new approach of teaching and learning in comparison with traditional forms of education.

2. The Measureserver unit is a core part of the ISES remote experiment used for data processing and communication among clients and ISES physical modules to control and monitor phenomena.

3. The intelligent data processing component is an important feature for the extracting, archiving and analyses of measured data and metadata generated by the ISES remote experiments in order to improve and optimize the educational process.

4. The future work will be aimed at the design of a new component intended for the phenomena simulations, data evaluation and analysis.

\section{REFERENCES}

[1] ZEMAN, P. Software environment for integration of measured data from remote laboratory and simulation. Ostrava. VŠB-Technical University of Ostrava, Czech Republic, 2012.

[2] ZEMAN, P. Software environment for control of remote experiments. Ostrava: VŠB-Technical University of Ostrava, Czech Republic, 2011.

[3] KRBEČEK, M. ISES remote experiments. Zlín. UTB in Zlín, Faculty of Applied Informatics, Czech Republic, 2015.

[4] KRBEČEK, M., F. SCHAUER and F. LUSTIG, Easy Remote ISES - Development Environment Remote Experiments, Innovations 2013. Potomac, USA, 2013, pp. 81-100.

[5] KRBEČEK, M., F. SCHAUER and K. VLČEK. Communication Requirements of Laboratory Management System. In: Latest Trends on Systems - Volume II: Proceedings of the 18th International Conference on Systems, 2014, p. 686-691. ISBN 978-1-61804-244-6. Available: http://www.europment.org/library/2014/santorini/bypaper/SYSTE MS/SYSTEMS2-56.pdf
[6] LUSTIG, F. Internet School Experimental System ISES [online]. Prague, Czech Republic, 2009 [cit. 2014-06-05]. Available: http://www.ises.info/index.php/en

[7] HAMID, R., and S. A. MOHAMMED. 2010. Remote Access Laboratory System for Material Technology Laboratory Work. In International Conference on Engineering Education - Proceedings, 311-16. Available: http://www.scopus.com/inward/record.url?eid=2-s2.079958730131\&partnerID=tZOtx3y1

[8] DRIGAS, A. S., J. VRETTAROS, L. G. KOUKIANAKIS, and J. G. GLENTZES. 2006. A Virtual Lab and E-Learning System for Renewable Energy Sources. WSEAS Transactions on Computers 5 (2): 337-41. Available: http://www.scopus.com/inward/record.url?eid=2-s2.033645137921\&partnerID=tZOtx3y1

[9] LEWIS, Forbes. Educational Materials: Recursive Descent Parsing [online]. Lexington, Kentucky, USA: University of Kentucky, 2012 [cit. 2015-06-27]. Available: http://www.cs.engr.uky.edu/ lewis/essays/compilers/rec-des.html

\section{AUTHORS}

Michal Gerža is currently working toward the Ph.D. degree at the Tomas Bata University in Zlín, Faculty of Applied Informatics, Czech Republic, in the field of remote laboratories. He is a research assistant and programmer involved in the development of the ISES remote experiments, especially in the form of finite-state machines, fault signaling, diagnostic systems, process simulations and visualizations. He is the author of six publications in the field of informatics.

František Schauer received a M.S. degree in Electronics from the Brno University of Technology in 1963 and his Ph.D. degree in Solid State Physics from Prague University of Technology in 1978. In 1982 he was appointed Associate Professor and in 1988 Professor in Condensed Matter Physics at the Technical Academy in Brno, Czech Republic. In 1993-2002 he was with the Faculty of Chemistry, Brno University of Technology and since then he was with the Polymer Centre of the Faculty of Technology. At present he is with the Tomas Bata University in Zlín, Faculty of Applied Informatics, Czech Republic and Trnava University in Trnava, Faculty of Education, Slovak Republic.

His main activities are molecular organic electronics, electronic structure spectroscopy by electrochemical and charge injection methods and computer assisted experiments. He is the author of about 200 papers in both Solid state physics, Education practice and Remote laboratories with about 350 SCI citations.

The paper was published thanks to the Grant of Internal Agency of the Tomas Bata University No. IGA/FAI/2015/013, and also partially by the Grant of Kega Agency as projects No. 011TTU-4/2012 and 020TTU4/2013 and the Grant of APVV Agency project No. APVV 0096-11. Submitted 31 Jnuary 2016. Published as resubmitted by the authors 28 February 2016. 